\title{
Potential of whole genome sequencing in the assessment of sensitivity of clinical isolate $M$. tuberculosis to antibiotics
}

\author{
Olga Berdyugina \\ IIP RAS, Ekaterinburg, Russia \\ berolga73@rambler.ru
}

\begin{abstract}
To study the potential of whole genome sequencing in assessing the sensitivity of the clinical isolate of M.tuberculosis is an important research task. The data obtained can be used when choosing antibiotics in the treatment of a patient.
\end{abstract}

Keywords - whole genome sequencing, M.tuberculosis, antibiotic

Motivation and aim

Motivation

Genomic research in phthisiology allows you to get closer to solving complex scientific and practical problems. The study of molecular genetic factors of multi drugresistant (MDR) and extensively drug resistant (XDR) of M.tuberculosis is an important area of research.

Aim

The aim of this work was to study the potential of whole genome sequencing in assessing the sensitivity of the clinical isolate of M.tuberculosis to antibiotics.

\section{Methods}

Clinical and radiological evaluation of the patient was performed upon admission to a medical institution, as well as at the stages of treatment, in accordance with the requirements of providing medical care to patients with pulmonary tuberculosis. The nature of tissue reactions was established by pathomorphological studies. A study was carried out on the lung resect with staining according to Van Gieson and Weigert. The nucleotide sequence of the clinical isolate of M.tuberculosis was studied by the method of genome sequencing, which is based on the principle of research by synthesis (pyrosequencing) using the GS Junior instrument (Roche, Switzerland). In addition, the following were used: thermal cycler for amplification of nucleic acids C1000 Touch with CFX96 module, Flash Gel Dock electrophoresis system, handheld fluorimeter Quanti-Fluor-ST, DynaMag TM magnetic tripod, GS Junior Seq Reagents and Enzymes reagents, GS Junior Sequencing Buffers, GS Junior Packing Beads \& Supplement CB, GS Junior Control Bead Kit, GS Junior Maintenance Wash Kit, GS Junior PicoTiterPlate Kit. Work with the data obtained as a result of the study included the use of comparative analysis involving information from international databases, and the use of nonparametric methods.

\section{Results}

Sequencing of the clinical isolate of M.tuberculosis from a patient with fibro-cavernous pulmonary tuberculosis was performed. The diagnosis is confirmed by culture growth during bacteriological examination. Concomitant pathology was diabetes. When a patient was admitted to the hospital, drug resistance to rifadin, tubazide, ethambutol, streptomycin was revealed, which developed three years after the diagnosis and irregular intake of prescribed anti-TB drugs. The length of hospital stay was 147 days. Treatment included valvular bronchial blockage, pneumoperitoneum, a course of capreomycin, pyrazinamide, cycloserine, ofloxacin, and paraaminosalicylic acid. The result of treatment is improvement.

Sequencing of the clinical isolate of M.tuberculosis from this patient was carried out in two stages. This was due to the fact that the molecular genetic structure of M.tuberculosis is characterized by the presence of a large number of triple G-C bonds, which leads to difficulties in their cleavage during the reaction.

As a result of the work carried out at one of the intermediate stages, namely, at the stage of evaluating the result of emulsion PCR, after the saturation of particles for sequencing was carried out, it was found that the obtained number of particles is insufficient to identify the result and there is a high risk of ineffective research.

The start of the re-study with a change in the ratio of the starting reagents led to the proper analysis result.

The result of the work was the identified nucleotide sequence of the clinical isolate of M.tuberculosis belonging to an infected patient.

The genotype of the studied M.tuberculosis isolate consisted of 4.25 million bp. There were 1060 large contigs, 4006 medium contigs. The largest contig was 39 thousand bp in length. The average reading length of the fragments was 502 nucleotides. It was aligned $95,21 \%$ of all fragments obtained. The minimum coverage of the fragments according to the results of the work was to be at least 4 , in our study it was 15 , which confirms the high quality of the study, as well as the high reliability of the data.

To study the information obtained, we used international databases (ncbi, rast, blast) containing previously sequenced sequences of M.tuberculosis, including data on at least 15 groups of M.tuberculosis, containing genomes from 4.20 to 4.54 nucleotide base pairs, that encode from 3739 to 4258 genes.

A comparison of our own data with the results of international databases of M.tuberculosis strains made it possible to establish that the studied clinical isolate had genes, encoding penicillin-binding proteins. The isolate was sensitive to penicillin.

Thus, the results of whole genome sequencing in assessing the sensitivity of clinical isolates of M.tuberculosis to antibiotics can be used in the selection of patient therapy.

\section{ACKNOWLEDGMENT}

This work was carried out as part of the state assignment of the Institute of Immunology and Physiology, Ural Branch of the Russian Academy of Sciences (topic No. AAAA-A18118020590108-7). 